\title{
Best Evidence: Comments on Meta-Analysis of Coiling versus Clipping
}

W e live in a world where evidence-based practice is increasingly demanded by health care purchasers and patients alike. The highest level of evidence is provided by multiple adequate-sized randomized controlled trials, especially a meta-analysis of such randomized controlled trials. The current study provides this for the first time. In an ideal situation, such an analysis will be undertaken on the individual patient datasets. Although not undertaken at the individual patient data level, this metaanalysis is important because it combines the data of the Barrow Ruptured Aneurysm Trial (BRAT) ${ }^{1}$ with the 2 previous randomized controlled trials available in this field, in particular, the large International Subarachnoid Aneurysm Trial (ISAT) trial. ${ }^{2}$

The 2 main criticisms of ISAT were that it only enrolled $20 \%$ of the eligible population and that the neurosurgical centers that partook in the study were not specialized neurovascular centers. Therefore, surgical clipping was often performed by junior neurosurgeons. ${ }^{3}$

In BRAT, all patients were treated at a highly experienced and specialized neurovascular center; nearly $70 \%$ of eligible patients were enrolled; and the surgery was performed or supervised by Robert Spetzler, MD, the doyen of modern American neurovascular surgeons. Thus, it was perhaps a bit of a surprise that the results of BRAT and ISAT were almost identical. In our opinion, this unequivocally counters those 2 major criticisms of ISAT.

It is interesting to note that no rebleeds occurred in the coiling group of BRAT. This finding probably reflects the improved equipment and techniques that are now available for coiling (similar low rates of rebleed have been documented in the recent Hydrocoil Endovascular aneurysm occLusion and Packing Study (HELPS $)^{4}$ and Cerecyte trials. ${ }^{5}$ It also adds to the growing evidence that postcoiling medium-term aneurysm durability is not a clinically significant problem.

The BRAT has garnered some criticism. First, it does not conform to the Consolidated Standards of Reporting Trials criteria for a scientifically conducted randomized controlled trial. Second, the protocol has never been published. Third, 97 of the 500 patients were not followed up at 1 year, and the data relating to these lost patients are not available. Finally, the randomization process was not random, which may have led to significant bias in the study.

The authors note the failings of the BRAT but argue that because all of the trials analyzed produced consistent results, the methodologic differences do not negate the validity of their metaanalysis. This cannot be easily dismissed.

A study should really only be included in a meta-analysis if it fulfills the criteria set down in the methods section of that metaanalysis, which, in this case, is that the study is a true randomized controlled trial. However, the authors state that true random allocation did not take place in BRAT; therefore, they have been a little disingenuous. Perhaps a better way to approach this problem might have been for the authors to simply state a caveat; that is, in the BRAT, the method of treatment allocation was not random but the method of treatment allocation, chosen for pragmatic real-life practice reasons, was unlikely to have caused significant bias to the overall outcome.

So far, only 1 randomized controlled trial (ISAT) has looked at other important outcome measures such as the incidence of psychological trauma and seizures. It is hoped that BRAT will, in due course, produce these data also, as these factors do need to be considered when treatment of an individual patient is being selected. A captain of industry may not be the best candidate for clipping if he or she harbors an aneurysm of the anterior communicating artery because of the evidence that executive function may be damaged - there is also the significantly increased risk for epilepsy. ${ }^{6}$ Also, wide-neck anterior communicating artery aneurysms have long been known for their risk for amnesia from clipping of important perforators. ${ }^{7}$

The meta-analysis concludes that unequivocal evidence exists that the results of coiling are superior to those of clipping in the treatment of aneurysms. We concur and believe that this statement, which is based on unequivocal level I scientific evidence, should make it mandatory that prior to any neurosurgical clipping of a ruptured aneurysm, a formal opinion is obtained from a fully trained endovascular specialist as to the feasibility and risks of coiling. Medicolegal considerations would make recording of these discussions clearly in the medical record a given. 


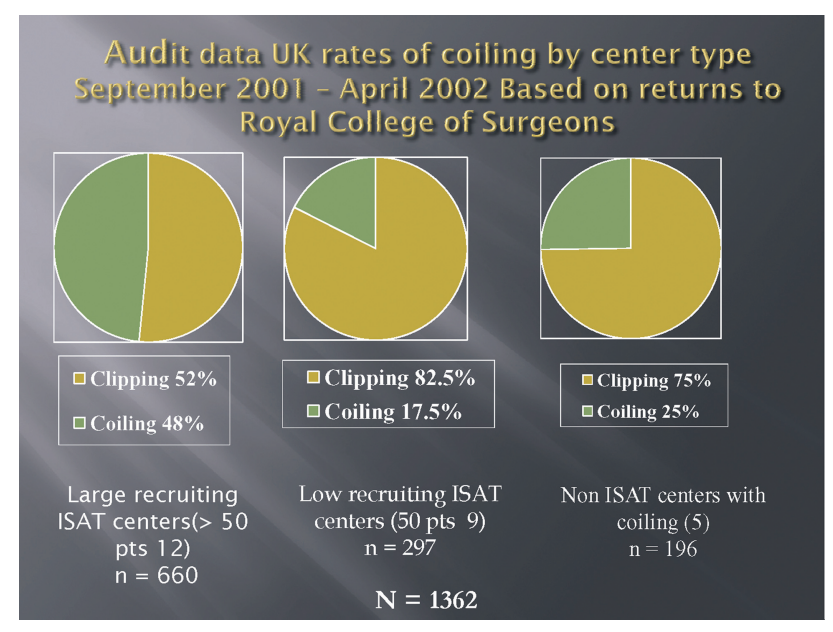

FIG 1. Rates of coiling and clipping in UK centers before ISAT data published. Data from the Society of British Neurological Surgeons National Audit.

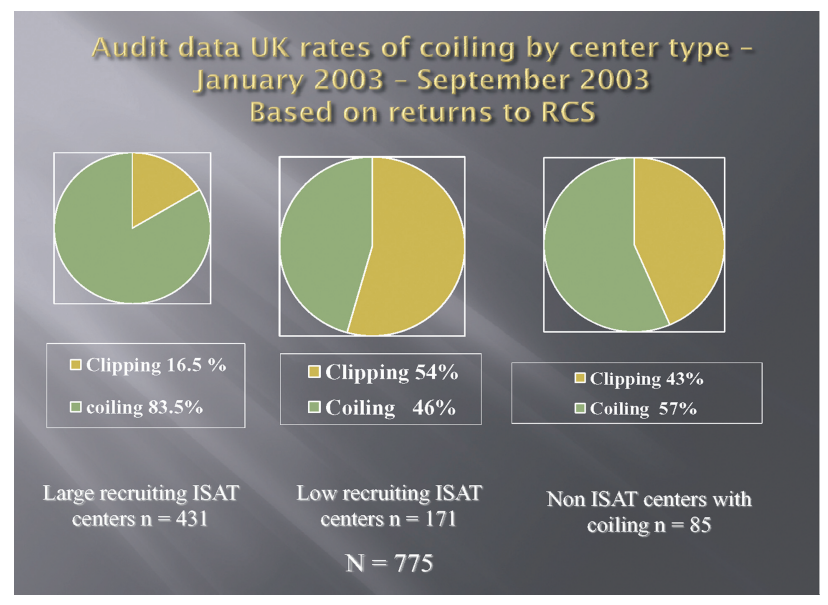

FIG 2. The Impact of the ISAT Trial data (released April 2002). Coiling rates in the large recruiting centers changed from $48 \%$ to $85 \%$ in the 6 months post data release.

Therefore, if coiling is the way to go for most aneurysms (where it is technically feasible), wither neurosurgical clipping?

An audit of the centers that participated in ISAT postpublication demonstrated that these centers mostly coiled $80 \%-85 \%$ of all aneurysms within months of ISAT publication [personal communication with A. Molyneux, September 2009] (Figs 1 and 2). Improved angiographic imaging and newer devices such as flowdiverting stents and intrasaccular flow-diverting devices will likely push that figure to roughly $90 \%$ in many centers. Thus, maintaining high-quality surgical clipping services will become a problem, particularly when there are so few cases for experienced neurosurgeons, let alone the neurosurgical trainees. Along with increasing evidence on the relationship between hospital/operator volumes and clinical outcomes, ${ }^{8}$ the inevitable conclusion is that concentration of vascular neurosurgery into large-volume centers is both desirable and inevitable. The minimal catchment population to support such a setup is debatable but probably is in excess of 4 million. Any such process of surgical concentration, of course, also affects the provision of neuroendovascular services. We are all in this together.

\section{REFERENCES}

1. McDougall CG, Spetzler RF, Zabramski JM, et al. The Barrow Ruptured Aneurysm Trial. J Neurosurg 2012;116:135-44

2. Molyneux A, Kerr R, Stratton I, et al. International Subarachnoid Aneurysm Trial (ISAT) of neurosurgical clipping versus endovascular coiling in 2143 patients with ruptured intracranial aneurysms: a randomised trial. Lancet 2002;360:1267-74

3. Sellar R, Whittle I. The ISAT trial. Lancet 2003;361:423-33; author reply 433

4. White PM, Lewis SC, Gholkar A, et al. Hydrogel-coated coils versus bare platinum coils for the endovascular treatment of intracranial aneurysms (HELPS): a randomised controlled trial. Lancet 2011; 377:1655-62

5. Coley S, Sneade M, Clarke A, et al. Cerecyte coil trial: procedural safety and clinical outcomes in patients with ruptured and unruptured intracranial aneurysms. AJNR Am J Neuroradiol 2012;33: $474-80$

6. Scott RB, Eccles F, Molyneux AJ, et al. Improved cognitive outcomes with endovascular coiling of ruptured intracranial aneurysms: neuropsychological outcomes from the International Subarachnoid Aneurysm Trial (ISAT). Stroke 2010;41:1743-47

7. Gade A. Amnesia after operations on aneurysms of the anterior communicating artery. Surg Neurol 1982;18:46-49

8. Bardach NS, Zhao S, Gress DR, et al. Association between subarachnoid hemorrhage outcomes and number of cases treated at California hospitals. Stroke 2002;33:1851-56

R.J. Sellar Division of Clinical Neurosciences University of Edinburgh Edinburgh, Scotland P. White Interventional and Diagnostic Neuroradiology University of Newcastle Newcastle upon Tyne, United Kingdom

http://dx.doi.org/10.3174/ajnr.A3404 